\title{
I-Voting System Based on Block Chain
}

\author{
Dr. D. Jayashree ${ }^{\mathrm{a}, 1}$, Dr.O. Pandithurai ${ }^{\mathrm{b}}, \mathrm{S}$ Yogeswari $^{\mathrm{c}}, \mathrm{K}$ B Swetha ${ }^{\mathrm{c}}$, A. Shalinic \\ ${ }^{a, b}$ Professor, Dept of CSE, Rajalakshmi Institute of Technology, Chennai, India \\ ${ }^{c} U G$ Scholar, Dept of CSE, Rajalakshmi Institute of Technology, Chennai, India
}

\begin{abstract}
The Blockchain based I-Voting uses a digital-currency analogy where in eligible voters can cast a ballot anonymously using a computing environment. BIV(Blockchain I-Voting) employs an encrypted key, smart biometrics and tamper proof real-time personal ID verification. Blockchain enable the creation of tamperproof audit trails for voting. In this generation of Technology there is an effect way for casting vote through online(Digital Voting) to make a public electoral process faster, cheaper, and more easier. In this generation it is being a compelling one in modern society which removes a power barrier between the elected candidate and the voter.
\end{abstract}

Keyword. Voting, BIV(Blockchain I-Voting), Digital voting.

\section{Introduction}

In our Day to day life, voting plays a major role for each and every citizen. The leader whom we choose has more responsibilities to develop a country in a more democratic way. On those days there were several voting methods to choose a leader for a country, (i.e, Paper based method, Machine voting, Postal voting, Online voting). The first voting method in every country is paper based method voting. In this voting process they will go to the polling booths and then the voter will be provided with an envelope, in that envelope they write the name of the leader or symbol of the party to whom they like to vote, and they put the envelope into the ballot they wish to vote. , "ONLINE VOTING SYSTEM' is an online voting technique. In this system people who have citizenship and above age of 18 with any sex(His/Her) are eligible for voting through online without going to any physical polling station. This voting system became more useful and advantageous than all the previous voting methods or patterns, this became more user friendly, but there were some drawbacks and issues in using this voting system, in many country's they are not ready to use this voting system because of its drawbacks. The only one country using the online voting is Estonia. Our aim of doing this project is to reduce the difficulties of voting, hereby we can vote from wherever we can access and all the country's in the world wide should use this online voting process, for this we are going to give a [1], secured process of voting system like using blockchain, hashing technology. Comparing to early days the number of voters has

${ }^{1}$ Dr D.Jayashree, Dept of CSE, Rajalakshmi Institute of Technology,Chennai, India.

Email id: nambikan@yahoo.com. 
been increased as the population increased, due to this we like to introduce new voting system which is based on blockchain. A blockchain based voting system is being developed to replace the current ballot box based system that requires manual effort. Digital voting systems already exist, but they are not blockchain based.

\section{Background Study}

In [1], The author proposed a new online voting system with secure authentication by using Steagnographic and biometric features. Intially the voter has to enter a password during registration. Using timestamp and hashing, here the password is converted into secret message. Once the process has over, the secret message is stored in image using steganography. Following steps are involved for vote recording and casting module, After successful authentication, a ballot is displayed, Voter cast their vote by selecting one of the candidates, This vote is encrypted and stored in database located at voter recording and casting server. Atlast the vote for the particular candidate will be generated.

In [2], The author have discussed various voting system and their advantages and disadvantages. The primary goal of this paper is to make the voting system multipurpose and make it work multiplatform on any operating system. As there is exponentially growth in the number of Smartphone users, we may say majority of the world population will own a Smartphone in future. Thereby they proposed a system which will work on any operating system be it windows, android or IOS using the QR code and OTP(one time password)functionality embedded in them. In this system the user can create their own voting ballot.

In [3], The author proposed online voting system, which addresses some of these challenges. According to voter's personal preferences, it eliminates all hardwired restrictions on the possible assignments of points to different candidates. In order to protect the confidentiality of the votes before submission, each cast ballot will be encrypted using the exponential ElGamal cryptosystem. Furthermore, during voting the system ensures that proofs are stored and generated for each element in the cast ballot during voting.

\section{An Overview Of Blockchain And Hashing}

In This Proposing Model there are four process for doing secure voting

i. Registration for voting

ii. Hashing Algorithm Technique

iii. Blockchain

\subsection{Registration for Voting}

The System Administrator Registers the voters on a special site of the system. These Registration process is done and visited only by him. Citizens who are registering for vote are expected to contact the system administrator for submitting the details. After submitting the credentials, the administrator will compare with their existing database details. 

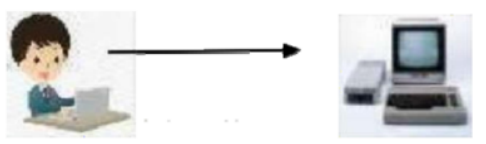

Figure 1. User and the Admin server

After registration the user will get a secret username and password, with that user will login and proceed the service provided like voting, checking results among others. The details are found to be invalid the voter is not allowed to register the vote.

\subsection{Hashing Algorithm Technique}

The main aim of hashing is to convert the given input of letters and numbers entered by the user into a output of fixed length in Encrypted format. A hash is build up with an algorithm and is essential to blockchain management in crypto currency (Digital Ledger for strong storing of database).

Personal health care information and medical record are stored in cloud. The above field must give more concentration to protect and give security in the cloud. Support

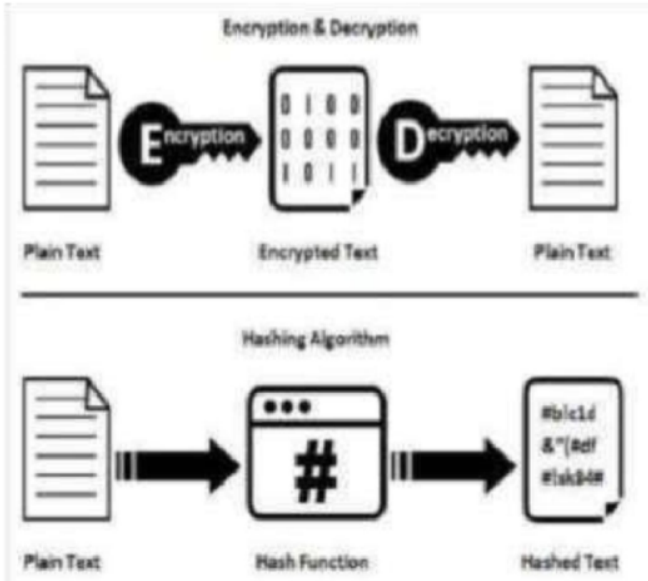

Figure 2. Hashing Algorithm

This process is referred as hashing the data. In general, When compared to the input data hash is much smaller, hence hash functions are sometimes called compression functions. Digest is a representation of larger data. In fig 2, It explains about the difference between Encryption, Decryption and the Hashing Algorithm, In encryption, while encrypting a plain text, plain text will be converted into a Encrypted text $(0 \mathrm{~s}$ and $1 \mathrm{~s})$ and if we want the plain text, by decrypting it we will get back the plain text. While applying hashing algorithm the input given by the user is converted into hashed text (ie. Undefined language). It is more secure and can't able to reuse by any other user, it will be accessible only for current user who registered and System Administrator. 


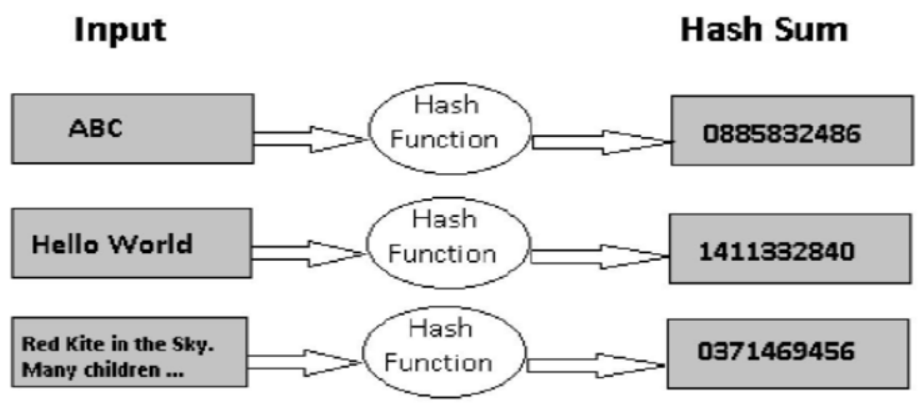

Figure 3. Conversion of hash function

\subsection{Blockchain}

Blockchain is a digital ledger. The technology draws its power from the peers or nodes - on its network to verify, process, and record all transactions across the system. This ledger is never stored, but rather exists on the "chain" supported by millions of nodes simultaneously. Thanks to encryption and decentralization, blockchain's database of transactions is incorruptible, and each record is easily verifiable. The network cannot be taken down or influenced by a single party because it doesn't exist in one place. This kind of system infrastructure is extremely useful for voting because a vote is a small piece of high- value data.

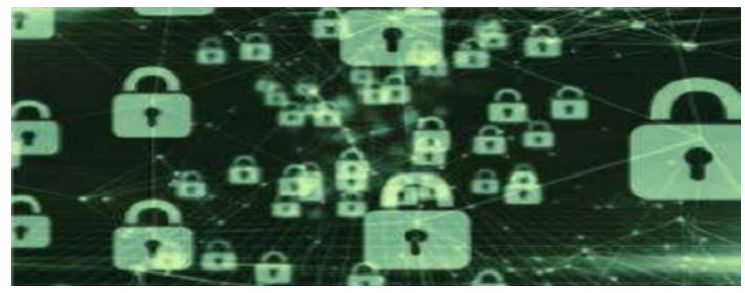

Figure 4. Secured Data

In Fig 4, Many problems discovered in these early attempts at online voting can be solved using blockchain. with the help of internet connection in the blockchain based ivoting system is highly secured, due to this hacker will not be able to affect other nodes. without revealing their identity or political preferences to the public voters can effectively submit their vote. Admin can count votes based on the user id, so that no fake votes can be created and tampering is impossible.

\section{Conclusion}

The proposed I-Voting system is based on the blockchain technology. Any registered voter will have the ability to vote using any device connected to the Internet. To make the input more secure we are using hash function and datas are locked using blockchain 
technique. So, nobody able to hack the user voting details. In this paper we analyse and review the cloud security basic, threats, machine learning technique and cloud security solution through machine learning technique. Finally we conclude machine learning technique attract the academic scholar and play a important role in sensing threats and occurrences.

\section{References}

[1] B.Smit Khaimar, P. Sanyasi Naidu, and Reena Kharat. On Secure Authentication for Online Voting System.Department of Computer Engineering, Pimpri Chinchwad College of Engineering, Pune, India. 2016.

[2] Dr.Z.A. Usmani, Mukesh Panigrahi, Kaif Patanwala, and Ajay Nair.On Multi-Purpose Platform Independent Online Voting System. Department of Computer Engineering, M. H. Sahoo Siddik College of Engineering, Mumbai, India, 2017.

[3] Xuechao Yang, Xun Yi, Surya Nepal, Andrei kelarev, and Fondling On A Secure Verifiable Ranked Choice Online Voting System, 2017.

[4] Jambhulkar S.M, Chakole B, and Pardhi R. A Secure Approach For Web based Internet Voting System Using Multiple Encryption. 2014.

[5] Lauretha Rura, Biji Issac, and Manas Kumar Haldar . Implementation and Evaluation of Stegnography Based on Online Voting System.2016.

[6] Himanshu Agarwal, G.N.Pandey . Online Voting System For India Based On AADHAAR ID. 2013.

[7] Sunita Patil, Amish Bansal, Vaibhavi Pujari, Utkarsh Raina and Roushan Kumar . E-Smart Voting System with Secure Data Identification using Cryptography. 2018.

[8] Rumeysa Bulut, Alperen Kantarci, Safa Keskin, and Serif Bahtiyar . Blockchain-Based Electronic Voting System for Elections in Turkey. 2019.

[9] Stradiotto, C.R.K. Zotti, A.I. Bueno, and S.P.M. Hoeschl .Web 2.0 e-voting system using android platform. 2010.

[10] R.Bhuvanapriya, S.Rozil Banu, T. Sivapriya and V.K.G. Kalaiselvi .Smart Voting .2017.

[11] VDAK, S. Sharmila, Abhishek Kumar, A. K. Bashir, Mamoon Rashid, Sachin Kumar Gupta \& Waleed S. Alnumay .A novel solution for finding postpartum haemorrhage using fuzzy neural techniques. Neural Computing and Applications (2021) (https://doi.org/10.1007/s00521-020-05683-z)

[12] AnkitKumar,VijayakumarVaradarajan,AbhishekKumar, PankajDadheech, Surendra SinghChoudhary, AKumar, B.K.Panigrahi, Kalyana C.Veluvolug . Black hole attack detection in vehicular ad-hoc network using secure AODV routing algorithm.Microprocessors and Microsystems, In Press,(https://doi.org/10.1016/j.micpro.2020.103352)

[13] VDAKumar.A Cognitive Model for Adopting ITIL Framework to Improve IT Services in Indian IT Industries.Journal of Intelligent Fuzzy Systems. (DOI: 10.3233/JIFS-189131)

[14] V.D.Ambeth Kumar.Efficient Data Transfer in Edge Envisioned Environment using Artificial Intelligence based Edge Node Algorithm. Transactions on Emerging Telecommunications Technologies , (DOI: 10.1002/ett.4110)

[15] V. D. Ambeth Kumar,S. Malathi,Abhishek Kumar,Prakash M and Kalyana C. Veluvolu.Active Volume Control in Smart Phones Based on User Activity and Ambient Noise.Sensors 2020, 20(15), 4117; https://doi.org/10.3390/s20154117

[16] Ambeth Kumar et.al.,.Exploration of an innovative geometric parameter based on performance enhancement for foot print recognition.Journal of Intelligent and Fuzzy System , vol. 38, no. 2, pp. 2181-2196, 2020.

[17] B. Aravindh; V.D.Ambeth Kumar; G. Harish; V. Siddartth, “ A novel graphical authentication system for secure banking systems", IEEE (ICSTM), Pages: 177-183, 2-4 Aug. 2017, DOI: 10.1109/ICSTM.2017.8089147

[18] K. Sabarinathan et.al ., " Machine Maintenance Using Augmented Reality”, 3rd International Conference on Communication and Electronics Systems (ICCES), 2018. (DOI: 10.1109/CESYS.2018.8723900)

[19] B. Aravindh; V.D.Ambeth Kumar; G. Harish; V. Siddartth, “ A novel graphical authentication system for secure banking systems”, IEEE (ICSTM), Pages: $177-183$, 2-4 Aug. 2017, DOI: 10.1109/ICSTM.2017.8089147 
[20] V.D.Ambeth Kumar et.al," "An Efficient Security System for Data base Management from Illegal Access, IEEE International Conference on Wireless Communications, Signal Processing and Networking, 2016. DOI: 10.1109/WiSPNET.2016.7566252 